\title{
Pulmonary Rehabilitation in Patients with Respiratory Disease
}

Furqan Shoaib Siddiqi, M.D. ${ }^{1}$, Said Chaaban, M.D. ${ }^{2}$, Erin Petersen, M.S.N., A.P.R.N. ${ }^{3}$, K James Kallail, Ph.D. ${ }^{2}$, Mary Hope, B.H.S., A.R.T., R.R.T., C.P.F.T. ${ }^{3}$,

Daniel Nichols, A.R.T., R.R.T., A.E.C. ${ }^{3}$

${ }^{1}$ University of Florida College of Medicine, Department of Medicine, Division of Pulmonary, Critical Care, and Sleep Medicine, Jacksonville, FL

${ }^{2}$ University of Kansas School of Medicine-Wichita

Department of Internal Medicine, Wichita, KS

${ }^{3}$ Via Christi Hospital on St. Francis, Pulmonary Rehabilitation, Wichita, KS

\begin{abstract}
Background. Limited evidence suggests that pulmonary rehabilitation be included in the management of restrictive lung diseases. The purpose of this study was to document pulmonary rehabilitation outcomes in patients with respiratory diseases other than chronic obstructive pulmonary disease (COPD).

Methods. Clinical outcomes of 31patients with respiratory diseases other than COPD and 190 patients with COPD, seen over a 35-month period, were reviewed retrospectively. Patients were evaluated for a 6-minute walk, arm curl strength, chair stand strength, the St. George's Respiratory Questionnaire (SGRQ) total score, SGRQ symptom scores, SGRQ activity levels, and SGRQ impact of respiratory illness on the patient's life. Outcome measures were obtained before the start of pulmonary rehabilitation and after a minimum of nine therapy visits.

Results. Pre- and post-rehabilitation changes in the 6-minute walk, arm curl strength, chair stand strength, the St. George's Respiratory Questionnaire (SGRQ) total score, SGRQ symptom scores, SGRQ activity levels, and SGRQ impact scores improved significantly for both groups. However, non-COPD patients achieved significantly higher mean SGRQ impact scores and arm curl strength than patients with COPD.
\end{abstract}

Conclusions. Pulmonary rehabilitation should be recommended for all patients with respiratory disease, not only those with COPD.

KS J Med 2014; 7(1):6-10.

\section{Introduction}

Many patients with obstructive lung diseases have activity limitations and deconditioning as a result of their poor lung function and dyspnea. ${ }^{1}$ In 2000, the American Thoracic Society issued a consensus statement supporting pulmonary rehabilitation in idiopathic pulmonary fibrosis (IPF) patients. ${ }^{2}$ In 2007, the American College of Chest Physicians and the American Association of Cardiovascular and Pulmonary Rehabilitation published a Grade $1 \mathrm{~B}$ evidence of recommendation for pulmonary rehabilitation $(\mathrm{PR})$ in patients with chronic lung diseases other than COPD. $^{2}$

Further study is needed to understand the benefits of PR in non-COPD patients. Limited evidence suggests that PR should be added within the management algorithm of restrictive lung diseases to reduce morbidity and lower the cost burden. The purpose of this study was to document the outcomes of PR in patients with respiratory diseases other than COPD and compare them to those with COPD. 


\section{Methods}

A retrospective review of $\mathrm{PR}$ outcomes was conducted in patients with respiratory disease other than COPD who were enrolled in a hospital-based pulmonary rehabilitation program between January 2009 and November 2011. These outcomes were compared to patients with COPD seen in the same program over the study period. Subjects completed a minimum of nine therapy sessions and were between the ages of 18 to 75 . Exclusion criteria included recent myocardial infarction, decompensated heart failure, terminal cancer, disabling cardiovascular accidents, dementia, alcoholism, pregnancy, prisoners, and age less than 18 or greater than 75 .

An order from a licensed physician was required for evaluation and treatment. Each subject had an initial evaluation visit performed by a respiratory therapist or registered nurse to verify the patient was an appropriate candidate for pulmonary rehabilitation. Baseline outcome measures were obtained at that visit.

The 6-minute walk evaluation was completed according to American Thoracic Society Guidelines. ${ }^{3}$ An arm curl test was performed using a bicep curl movement with a hand weight. Men used an 8-pound weight; women used a 5-pound weight. The maximum number of full bicep curls completed in 30 seconds was recorded. The chair stand test was performed using a designated chair. Patients were instructed to rise from seated position to a full stand position, without using arm swing or push for assistance and maximum number of sit to stand movements in 30 seconds were recorded.

The patient's quality of life was assessed using the St. George's Respiratory Questionnaire (SGRQ). It is a 16-item questionnaire measuring health impairment in patients with lung disease. The questionnaire assessed patients' 1-month recall of respiratory symptoms, activity disturbance, and impact of respiratory status on their lives.

Data from these variables as well as history and physical exam were used to create an individualized treatment plan for approval by the patient's referring physician and the PR medical director. After the initial evaluation, patients returned for twice weekly visits for breathing retraining, education to improve self-management of lung disease, exercise training, and social support. After finishing a minimum nine sessions of pulmonary rehabilitation, measurements were repeated to compare with the baseline. The comparison of patient variables before and after the program provided the data for this study.

This study was approved by the institutional review boards at Via Christi Health and the University of Kansas School of Medicine-Wichita.

Data were analyzed between groups for each outcome variable using an analysis of variance for independent samples. Data were analyzed within groups for each outcome variable using a repeated measures Student t. Demographics were analyzed by Student $t$ tests.

\section{Results}

Thirty-one patients with respiratory disease other than COPD and 190 patients with COPD met study criteria. Diagnoses in the non-COPD group included bronchiectasis $(n=1)$, pulmonary fibrosis $(n=6)$, interstitial lung disease (ILD; $\mathrm{n}=8$ ), pulmonary hypertension $(n=6)$, sarcoidosis $(\mathrm{n}=5)$, asthma $(\mathrm{n}=2)$, cystic fibrosis $(\mathrm{n}=$ $1)$, lung cancer $(\mathrm{n}=1)$, and Wegner's granulo-matosis $(n=1)$. Mean age in the non-COPD group was 63 years and was significantly younger than the 70 years in the COPD group $(\mathrm{p}<0.001)$. 
Non-COPD and COPD groups independently showed significant improvement with rehabilitation on all outcomes measures (Tables 1 and 2). The non-COPD group revealed better combined
SGRQ impact scores (45.1 vs 36.8; p < $0.001)$ and arm curl strength scores (17.6 vs $14.2 ; \mathrm{p}<0.001)$ than the COPD group. No other group differences were observed.

Table 1. Mean pre- and post-pulmonary rehabilitation assessments in patients with non-COPD diagnosis.

\begin{tabular}{|l|c|c|c|}
\hline Tests & Pre-Rehabilitation & Post-Rehabilitation & p value \\
\hline SGRQ total score & 58 & 50 & $<0.05$ \\
\hline SGRQ symptom score & 53 & 45 & $<0.01$ \\
\hline SGRQ impact score & 48 & 40 & $<0.05$ \\
\hline SGRQ activity score & 76 & 68 & $<0.01$ \\
\hline 6-minute walk & 245 & 285 & $<0.01$ \\
\hline Chair stand strength & 8 & 9 & $<0.05$ \\
\hline Arm curl strength & 16 & 19 & $<0.001$ \\
\hline
\end{tabular}

Table 2. Mean pre- and post-pulmonary rehabilitation assessments in patients with COPD.

\begin{tabular}{|l|c|c|c|}
\hline Tests & Pre-Rehabilitation & Post-Rehabilitation & p-value \\
\hline SGRQ total score & 54 & 44 & $<0.0001$ \\
\hline SGRQ symptom score & 58 & 46 & $<0.0001$ \\
\hline SGRQ impact score & 43 & 30 & $<0.0001$ \\
\hline SGRQ activity score & 73 & 65 & $<0.0001$ \\
\hline 6-minute walk & 265 & 311 & $<0.0001$ \\
\hline Chair stand strength & 7 & 9 & $<0.0001$ \\
\hline Arm curl strength & 12 & 16 & $<0.0001$ \\
\hline
\end{tabular}

\section{Discussion}

Pulmonary rehabilitation improved all measured outcomes in both groups of patients. PR was beneficial to patients with all types of respiratory disease, not only those with COPD. The younger group of non-COPD patients showed higher SGRQ impact scores and arm curl strength. These differences may be related to age or severity of disease. No group differences, however, were seen in any other outcome measures.

PR aids in improving a patient's functional status and controlling their symptoms, especially dyspnea (Grade 1A evidence) and fatigue. ${ }^{4}$ PR enlightens patients about their disease treatment options and improves their physical 
capabilities and capacities. Low self-esteem and quality of life usually is present secondary to physical disability thus worsening the patient's main symptom, shortness of breath. Anxiety also worsens dyspnea. On the other hand, depression, anxiety, and dyspnea worsens the underlying physical impairment. ${ }^{5}$ PR does not reverse the disease but reduces symptoms, disability, and mortality, resulting in a decrease in hospital stay and reduction in hospital admissions, thus lowering the cost burden on health care system. ${ }^{4}$

PR consists of patient assessment, exercise, dietary recommendations, and psychosocial support. ${ }^{4}$ Training of muscles of ambulation must be implemented within the program (Grade 1 A recommendation). Benefits are observed over 6 to 12 weeks, with longer programs producing more benefits. $^{3}$ Effects may last up to 18 months in COPD patients; ${ }^{6}$ no data are known for patients with other respiratory diseases.

Rehabilitation programs have been well developed for patients suffering from advanced COPD. It also has been used in non-COPD patients, mainly with interstitial lung diseases, cystic fibrosis, bronchiectasis, and thoracic cage abnormalities. ${ }^{4}$ No clinical recommendation is available with regards to

\section{References}

${ }^{1}$ Cambach W, Wagenaar RC, Koelman TW, van Keimpema AR, Kemper HC. The long-term effects of pulmonary rehabilitation in patients with asthma and chronic obstructive pulmonary disease: a research synthesis. Arch Phys Med Rehabil 1999; 80(1):103-111. PMID: 9915381.

2 Markovitz GH, Cooper CB. Rehabilitation in non-COPD: mechanisms of exercise limitation and pulmonary rehabilitation for patients with pulmonary fibrosis/restrictive lung disease. Chron Respir Dis 2010; 7(1): 47-60. PMID: 19880656.
PR in diseases other than COPD. All of the evidence for non-COPD programs was based on expert opinion. Treatment for these patients should be individualized. ${ }^{7}$

Growing evidence supports the need for PR in patients with Idiopathic Pulmonary Fibrosis (IPF). ${ }^{7,8} \mathrm{PR}$ in restrictive lung diseases has shown promising results. ${ }^{8}$ Foster and Thomas evaluated 32 patients with ILD, bronchiectasis, fibrothorax, thoracoplasty, and neuromuscular abnormalities and they concluded that the degree of benefit was equivalent to COPD patients. ${ }^{9}$ PR improves activity and health-related quality of life in patients with idiopathic pulmonary fibrosis. ${ }^{10}$ Further research is required to assess the optimal timing of PR and if there is a difference in benefit within subgroup of diseases. ${ }^{11}$

Limitation of the data exist with restrictive lung disease, and that is because of the heterogeneity of the pathophysiology of the diseases, thus leading to different mechanisms for exercise limitations. ${ }^{2}$ Our study showed that PR may be beneficial not only in patients with COPD, ILD, and IPF, but also in other pulmonary diseases. Further disease specific studies should establish standard protocols and guidelines for referral to PR in non-COPD diagnosis.

3 ATS Committee on Proficiency Standards for Clinical Pulmonary Function Laboratories. ATS Statement: Guidelines for the six-minute walk test. Am J Respir Crit Care Med 2002; 166(1):111-117. PMID: 12091180.

${ }^{4}$ Ries AL, Bauldoff GS, Carlin BW, et al. Pulmonary rehabilitation: Joint ACCP/ AACVPR Evidence-Based Clinical Practice Guidelines. Chest 2007; 131(5 Suppl):4S-42S. PMID: 17494825.

5 Ochmann U, Jörres RA, Nowak D. Longterm efficacy of pulmonary rehabilitation: a state-of-the-art review. J Cardiopulm 
Rehabil Prev 2012; 32(3):117-126. PMID: 22354015.

6 Troosters T, Gosselink R, Decramer M. Short- and long-term effects of outpatient rehabilitation in patients with chronic obstructive pulmonary disease: A randomized trial. Am J Med 2000; 109(3): 207-212. PMID: 10974183.

7 Varadi RG, Goldstein RS. Pulmonary rehabilitation for restrictive lung diseases. Chest 2010; 137(2):247-248. PMID: 20133284.

${ }^{8}$ Kagaya H, Takahashi H, Sugawara K, Kasai C, Kiyokawa N, Shioya T. Effective home-based pulmonary rehabilitation in patients with restrictive lung diseases. Tohoku J Exp Med 2009; 218(3):215-219. PMID: 19561392.

${ }^{9}$ Foster S, Thomas HM $3^{\text {rd }}$. Pulmonary rehabilitation in lung disease other than chronic obstructive pulmonary disease. Am Rev Respir Dis 1990; 141(3):601-604. PMID: 2310092.

${ }^{10}$ Holland AE, Hill CJ, Conron M, Munro P, McDonald CF. Short term improvement in exercise capacity and symptoms following exercise training in interstitial lung disease. Thorax 2008; 63(6):549-554. PMID: 18245143.

${ }^{11}$ Mainguy V, Maltais F, Saey D, et al. Effects of a rehabilitation program on skeletal muscle function in idiopathic pulmonary arterial hypertension. J Cardiopulm Rehabil Prev 2010; 30(5): 319-323. PMID: 20410828.

Keywords: pulmonary rehabilitation, restrictive lung disease, interstitial lung disease, chronic obstructive pulmonary disease 